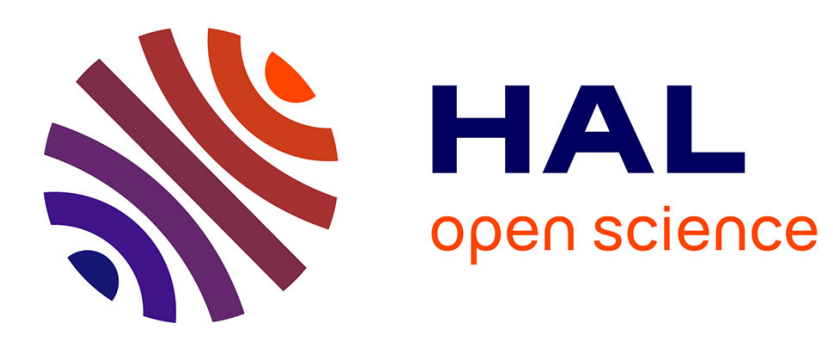

\title{
An improved shielded RF transmit coil for low-frequency NMR and MRI
}

Viacheslav V. Kuzmin, Christopher P. Bidinosti, Michael E. Hayden, Pierre-Jean Nacher

\section{- To cite this version:}

Viacheslav V. Kuzmin, Christopher P. Bidinosti, Michael E. Hayden, Pierre-Jean Nacher. An improved shielded RF transmit coil for low-frequency NMR and MRI. Journal of Magnetic Resonance, 2015, 256, pp.70-76. 10.1016/j.jmr.2015.05.001 . hal-01141760

\section{HAL Id: hal-01141760 \\ https://hal.science/hal-01141760}

Submitted on 13 Apr 2015

HAL is a multi-disciplinary open access archive for the deposit and dissemination of scientific research documents, whether they are published or not. The documents may come from teaching and research institutions in France or abroad, or from public or private research centers.
L'archive ouverte pluridisciplinaire HAL, est destinée au dépôt et à la diffusion de documents scientifiques de niveau recherche, publiés ou non, émanant des établissements d'enseignement et de recherche français ou étrangers, des laboratoires publics ou privés. 


\title{
An improved shielded RF transmit coil for low-frequency NMR and MRI
}

\author{
V.V. Kuzmin ${ }^{1}$, C.P. Bidinosti², M.E. Hayden ${ }^{3}$, and P.-J. Nacher*1 \\ ${ }^{1}$ Laboratoire Kastler Brossel, ENS-PSL Research University, CNRS, UPMC-Sorbonne Universités, Collège de France; 24 rue \\ Lhomond, F-75005 Paris, France. \\ ${ }^{2}$ Department of Physics, University of Winnipeg, Winnipeg, MB, Canada R3B 2E9. \\ ${ }^{3}$ Department of Physics, Simon Fraser University, Burnaby, BC, Canada V5A $1 S 6$.
}

\begin{abstract}
We describe an actively shielded cylindrical RF transmit coil producing a highly uniform internal field $( \pm 0.5 \%)$ over an extended volume and a strongly suppressed $(\div 20)$ external field. Direct field mapping and experimental checks using in-situ NMR and MRI of polarised ${ }^{3} \mathrm{He}$ at low temperature demonstrate performance consistent with numerical field computations.
\end{abstract}

\section{Introduction}

Obtaining highly uniform RF fields over extended volumes is mandatory for pulsed NMR and MRI experiments requiring accurate tipping angles. This requirement may occur, for instance, when series of fixed or variable smallangle flips are used in hyperpolarised samples. Typical $\mathrm{RF}$ resonator designs, such as the widely used birdcage [1] with or without passive screening, provide a choice of solutions at conventional NMR frequencies. Achieving good performance from a resonant structure becomes more challenging at low frequency, however [2]. In contrast, wire-wound RF coils, either untuned or tuned by separate capacitors, are suitable for low-frequency NMR (up to a few $\mathrm{MHz}$ at most, depending on coil size [3]).

Cylindrical saddle-shaped loops are convenient building blocks for designing RF coils with outstanding field uniformity (4] and references therein). However, the large RF field outside such coils may induce eddy currents in neighbouring conductors and therefore affect RF field uniformity. Moreover, eddy currents may result in significant heating of surrounding conductors, which must be avoided in low temperature experiments. The eddy currents can be actively reduced using a surrounding shield coil designed for this purpose 4 .

One of the coil-shield sets proposed in Ref. 4 has been used as an untuned transmit coil in NMR experiments performed at $87 \mathrm{kHz}$ on hyperpolarised liquid helium-3 [5]. It comprised five saddle pairs (the main coil in Fig. 1) and four loop pairs (the shield coil in Fig. 1), connected in series with opposite current flow directions. The homogeneity of this coaxial coil-shield set, while good for an MRI coil, was found to be insufficient for the demands of experiments involving a very large number of large-angle RF pulses.

We report here on accurate characterisations of the net

\footnotetext{
*nacher@lkb.ens.fr, corresponding author.
}
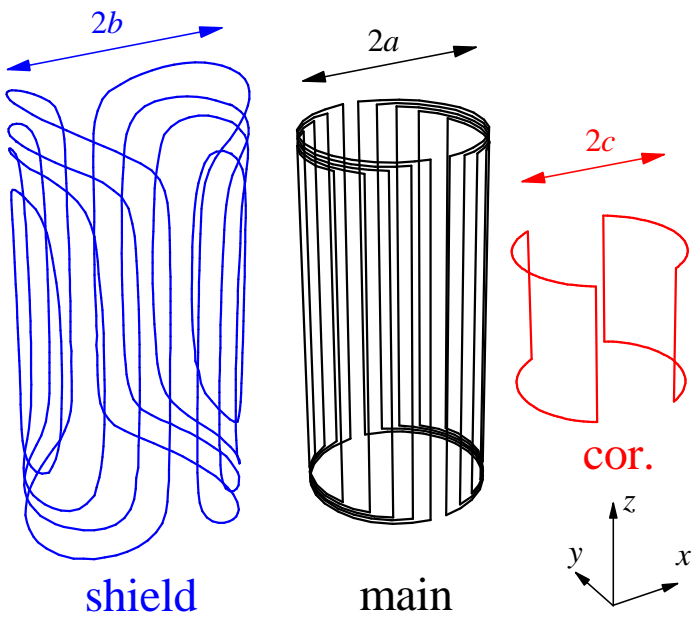

Figure 1: Set of coaxial coils displayed side by side for clarity. Centre: the main coil consists of five saddle pairs of 10 turns each, with diameter $2 a=25.8 \mathrm{~mm}$. The lengths of the saddle pairs could be equal 4 or slightly different, as displayed here (our experiment, App. A. Left: the outer shield coil consists of four loop pairs (10 turns each, diameter $2 b=32 \mathrm{~mm}$ ). Right: a correcting coil (a 2 turns saddle coil pair, diameter $2 c=24 \mathrm{~mm}$ ) fits inside the main coil. All loop and saddle pairs are connected in series by twisted pairs of wires (not shown). The shield length is 65 $\mathrm{mm}$; other dimensions are given in App. A. The uniform transverse field is along the $x$-axis at the coil centres.

RF uniformity of this coil set and on significant improvements of its performance through the introduction of an additional correcting coil. In particular, we demonstrate a ten-fold increase in uniformity without appreciable degradation of the shielding efficiency. The following section is devoted to numerical field map computations; the subsequent one describes experimental results obtained by direct field mapping at room temperature and using ded- 
icated NMR and MRI measurements at low temperature in the presence of nearby conductors. Details of the coil construction, the numerical evaluation of RF shielding efficiency, and the reconstruction of RF maps from MRI data are provided in Appendices.

\section{Computed field maps}

Numerical field computations of the Biot-Savart law over 3D matrices inside and outside the coil of Fig. 1 (modelled as 1500 straight current elements, with variable lengths suitably approximating the curved wires) were performed using an in-house Fortran program. Given our low-frequency operation and small coil size, the currents were assumed to be everywhere in-phase and static calculations were performed. Averages and variances of fields over selected regions of interest were used to assess levels of uniformity for the internal field (inside the main coil) and of shielding for the external field (outside the shield coil) for different coil configurations.

Figure 2 displays results of field calculations in the vicinity of the coil axis, for $x$ or $y$ up to $30 \%$ of the main coil radius, $a$. For our initial coil-shield set, a limited uniformity was found: the RF field amplitude $B_{1}$ increases with $|z|$ by up to $8 \%$ for $z / a=1$ (set of curves labelled "uncorrected"; see also Fig. 4a). Variations of the total field in transverse planes at fixed $z$ positions are smaller than those of the fields of the main or the shield coil and can usually be neglected. The shield coil provides an efficient shielding (see App. B but significantly degrades the internal field uniformity compared to that of the main coil over a total length of the order of the coil radius.

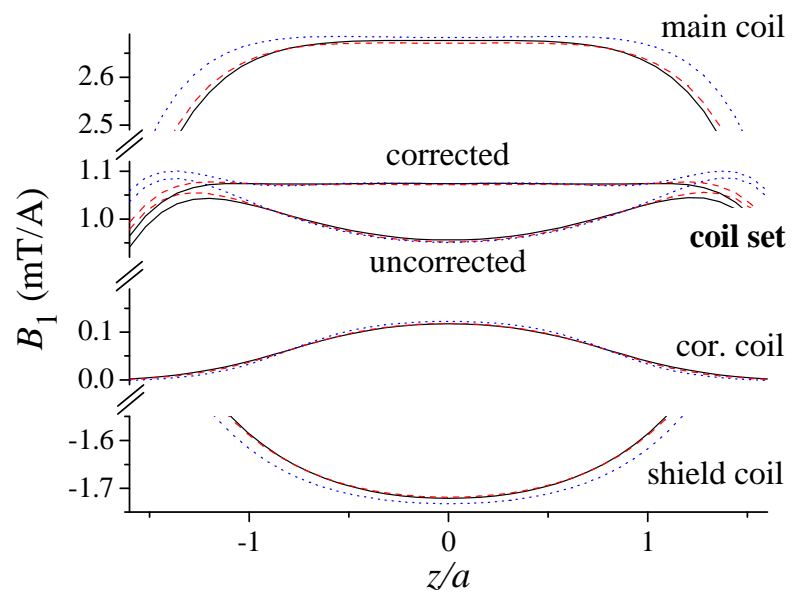

Figure 2: Top to bottom: computed fields generated by the main coil alone, the full set of coils ("corrected") or the main+shield set ("uncorrected"), the correcting coil, and the shield coil (the negative $B_{1}$ values result from the opposite current direction). The reduced position $z / a$ is scaled to the radius of the main coil. Solid (black) lines: values on the coil axis. Dotted (blue) lines: $y / a=0.3$. Dashed (red) lines: $x / a=0.3$.
Various changes of the coil shapes and the addition of a correcting coil have been numerically compared. Replacing cylinders by paraboloids for the main coil (with radius $a$ increasing with $z^{2}$ ) and/or the shield coil (with $b$ decreasing with $z^{2}$ ) can be used to reduce the variation of the total field with $z$ but significantly degrades the shielding efficiency. Using the additional correcting coil shown in Fig. 1 also restores good internal RF uniformity, with $B_{1}$ varying by less than $\pm 0.5 \%$ for $z / a \leq 1$ (set of curves labelled "corrected"; see also Fig. 4 b). The radius and length of the correcting coil were jointly adjusted to yield an optimal correction (the number of turns was fixed and a $60^{\circ}$ angle was chosen for better field homogeneity in the transverse plane). Compared to changes in the coil or shield shapes, the addition of this correcting coil better preserves a high shielding efficiency and is easier to implement experimentally. It is the approach examined in the remainder of this paper.

\section{Experimental characterisations}

\subsection{Methods}

RF field maps were directly probed along the coil axis at room temperature using a search coil operated at $50 \mathrm{kHz}$. They were also inferred from NMR experiments performed at low temperature on He samples. For the latter, we took advantage of the proportionality between the local RF field $B_{1}(z)$ and the local flip angle $\theta(z)$. Long RF pulses $(\Theta$ pulses $)$ were applied in order to induce large amplitude rotations of average angle $\Theta \gg 2 \pi$. Significant deviations of the local flip angle from the average flip angle were thus obtained and therefore RF inhomogeneities could be characterised with high sensitivity. ${ }^{3} \mathrm{He}$ gas was polarised by optical pumping at room temperature and transported through a $1 \mathrm{~mm}$ i.d. fill line to a $25 \mathrm{~mm}$ long, $4 \mathrm{~mm}$ i.d. cold glass cell [5]. NMR experiments were performed at $87 \mathrm{kHz}(2.7 \mathrm{mT})$ using a Tecmag Apollo console.

For initial experiments with the coil-shield set, the cold cell volume was centred on the coil and uniformly filled with $\sim 2$ mbar ${ }^{3} \mathrm{He}$ gas at $4.2 \mathrm{~K}$. Polarisation was achieved through gas diffusion along the fill line (the build-up time was $\sim 200 \mathrm{~s}$, the steady-state polarisation was $\sim 0.1$ ). In some experiments, an imaging technique was used to map the field produced by the RF coil; the cell temperature was lowered to $1.3 \mathrm{~K}$ to reduce the impact of diffusion which tends to erase spatial magnetisation inhomogeneities in the gaseous sample (cooling reduced the diffusion coefficient from $\sim 1 \mathrm{~cm}^{2} / \mathrm{s}$ to $\sim 0.4 \mathrm{~cm}^{2} / \mathrm{s}$ at the operating pressure [6]). Spin-echo data with a gradient $G$ applied along the coil axis were used to obtain $1 \mathrm{D}$ maps of the transverse magnetisation following a $\Theta$ pulse (Fig. 3 a). The whole sequence was performed with two values of $\Theta$ : $\Theta_{1}$ and $\Theta_{2}=\Theta_{1}+\pi / 2$. The value $\Theta_{1}=5 \times 2 \pi$ was chosen so as to yield a good sensitivity and avoid significant diffusion-induced averaging of magnetisation during the pulse. The two corresponding $1 \mathrm{D}$ images, $M_{1}(z)$ and 
$M_{2}(z)$, enabled us to reconstruct the RF field map $B_{1}(z)$. For a weak RF inhomogeneity, $\theta_{1}(z)$ could be obtained from $\tan \theta_{1}(z) \approx \Re\left[M_{1}(z) / M_{2}(z)\right]$ (see App. C, in which an exact method to reconstruct the RF field map is described). A global (volume-averaged) RF inhomogeneity measurement was also performed using a NMR sequence (Fig. 3b) consisting of initial and final RF pulses to evaluate initial and final magnetisations $(\alpha=\pi / 8$ or $\pi / 16$, depending on $\Theta$, and $\beta=\pi / 2$ ) and of an intermediate $\Theta$ pulse $(\Theta / 2 \pi$ ranging from $N=1$ to 60$)$. Crusher gradients were applied in order to prevent interferences between FID signals. The amplitudes of the two last signals in this sequence, $S_{\Theta}$ and $S_{\beta}$, were proportional to the transverse and longitudinal components of the average magnetisation, $\overline{M^{\prime}}$, remaining after the large rotation induced by the $\Theta$ pulse. The fractional surviving average magnetisation was thus $\overline{M^{\prime}} / M=\sqrt{S_{\Theta}^{2}+\left(S_{\beta}^{2} / \sin ^{2} \beta\right)} /\left(S_{\alpha} \cot \alpha\right)$.

For the experiments performed with the correcting coil, the cell was partly filled with ${ }^{3} \mathrm{He}-{ }^{4} \mathrm{He}$ liquid at $1.3 \mathrm{~K}$, and the coil was centred on the $10 \mathrm{~mm}$ high sample filling the lower part of the cell. Polarised ${ }^{3} \mathrm{He}$ gas was introduced through the fill line and dissolved in liquid ${ }^{4} \mathrm{He}$ to obtain a polarised sample (stopped flow after $10-30 \mathrm{~s}, T_{1} \geq 200 \mathrm{~s}$ ). The RF inhomogeneity was only globally assessed, using the sequence of Fig. $3 \mathrm{p}$ with $\alpha$ and $\beta$ chosen to combine high SNR and moderate magnetisation losses. The mea-

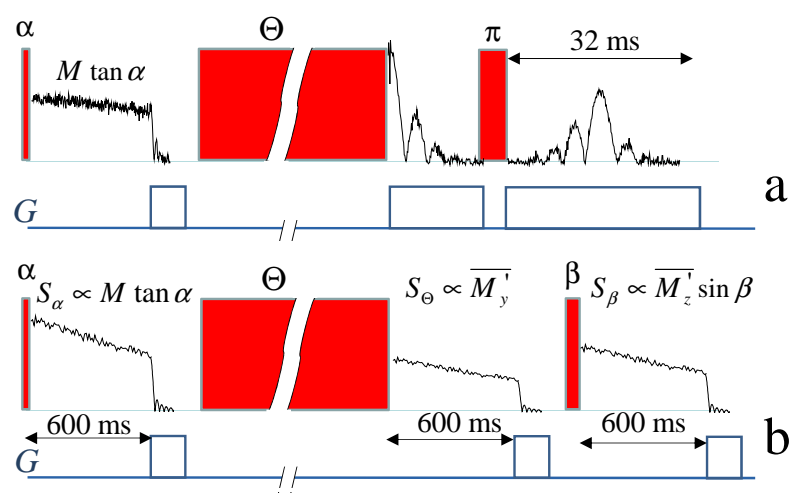

Figure 3: Typical amplitudes of complex quadrature signals obtained from polarised ${ }^{3}$ He samples. a: 1D imaging sequence used to evaluate the local value $\theta(z)$ of a rotation with average angle $\Theta \gg 2 \pi$. A composite $\pi$ pulse [7] was used for uniform flipping. A z-gradient $G=0.43 \mathrm{mT} / \mathrm{m}$ yielded a $2.3 \mathrm{~mm}$ nominal resolution. $\theta(z)$ was inferred from data recorded for two values of $\Theta$ (see text), scaled to the magnetisation $M$ remaining after the probing $\alpha=\pi / 20$ flip and crusher gradient. b: NMR sequence used to evaluate global rotation losses. The free induction decay (FID) signals were used to assess magnetisations $M$ (uniform) and $\overline{M^{\prime}}$ (volume-averaged) before and after the $\Theta$ pulse. Following a $\Theta$ rotation of exactly $N$ turns, the signal amplitude $S_{\Theta}$ would be zero. This was used to calibrate $\mathrm{RF}$ pulse amplitudes for $N=1$ ( $2 \pi$ pulse duration: $2.2 \mathrm{~ms}$ ). surements were repeated for different values of $\Theta$ on the same sample as long as enough magnetization remained.

\subsection{Results}

Figure 4 displays experimental results obtained with our initial coil-shield set (Fig. 4 a) and with the improved design including the correcting coil (Fig. 4b). Field maps were found to be unaffected by nearby metal elements, both at room and low temperatures, thanks to the good shielding efficiency. For both coil configurations, the measured field maps are in good agreement with the numerically computed ones. The expected improvement in field homogeneity is obtained with the addition of the correcting coil (note the five-fold change in the vertical scales).
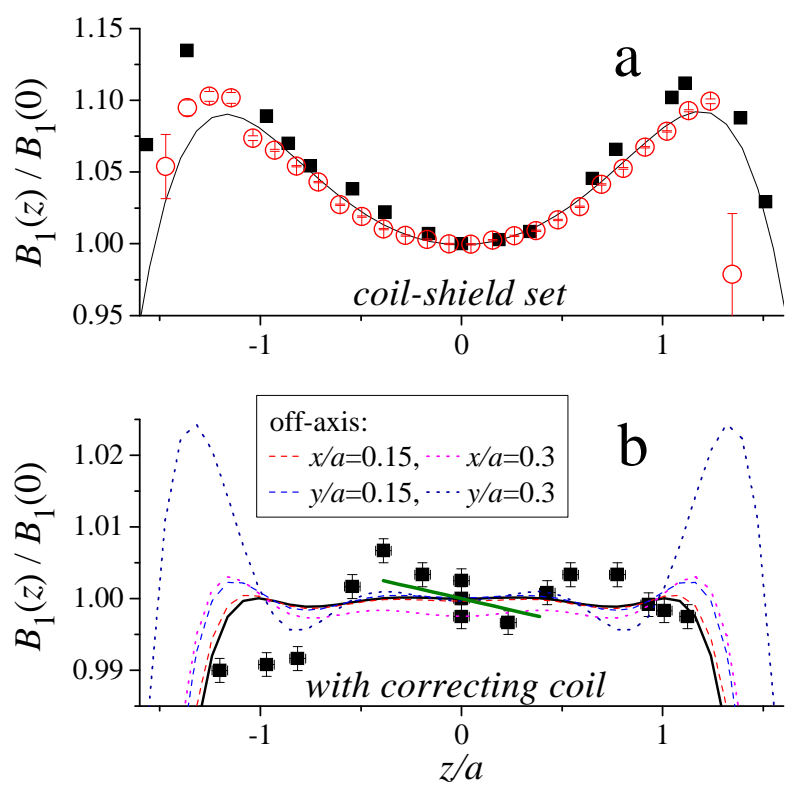

Figure 4: Relative field variations with $z / a$ for the initial coil-shield set (panel a) and with the addition of the correcting coil (panel b). Filled squares: data measured at room temperature with the search coil. Open circles: data inferred from 1D MRI measurements on ${ }^{3} \mathrm{He}$ gas. All lines are results of field computations (solid lines: on the axis; other lines: off-axis, see legend). The straight (green) line in panel b corresponds to $\nabla B_{1} / B_{1}=5 \times 10^{-3} \mathrm{~cm}^{-1}$ in a 10-mm-long sample (see text and discussion of Fig. 5)

Figure 5 displays the measured and computed average magnetisation amplitudes remaining after a $\Theta$ pulse. They were computed using a simple model: a set of vectors rotating around the $x$-axis with Rabi frequencies corresponding to the computed $B_{1}$ maps inside the samples (diffusion effects were neglected). With the initial coilshield set, the observed surviving average magnetisation (Fig. 5a) strongly decreases for $\Theta / 2 \pi$ exceeding a few turns. This is consistent with the expected dispersion of rotation angles across the $25 \mathrm{~mm}$ long sample. For a shorter sample (dotted line), the decrease would be sig- 
nificantly reduced due to the near- $z^{2}$ dependence of the field variation. With the correcting coil, the observed surviving average magnetisation (filled squares in Fig. 5b) was significantly larger but not as large as expected for a $10 \mathrm{~mm}$ long sample in the computed map of Fig. $4 \mathrm{~b}$ (the nearly horizontal dotted line in Fig. 5b). This is likely due to an imperfect relative positioning of the coils. Computations show that the field maps are rather insensitive to small changes in coil dimensions or positions, except for the linear term arising from the difference in $z^{2}$ variations of the fields from the shield coil and the correcting coil if their centres do not coincide. This yields a relative gradient in the total field of the order of $10^{-2} \mathrm{~cm}^{-1}$ per $\mathrm{mm}$ of $z$-offset in coil positions. The experimental data in Fig. 5b are thus consistent with expectations assuming an offset of the correcting coil of half a millimetre, which corresponds to the precision of the coil set assembly.
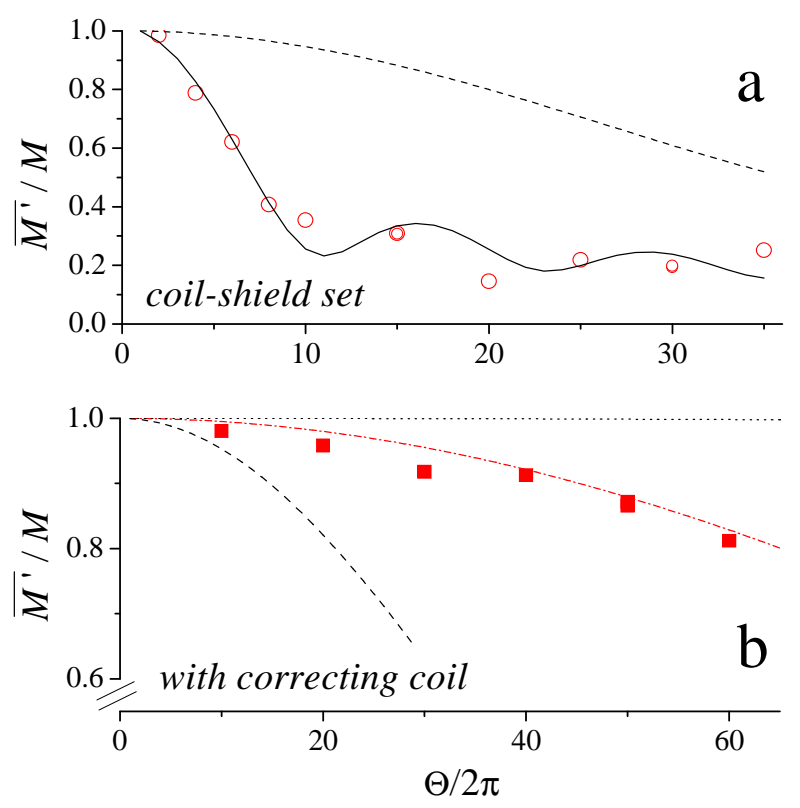

Figure 5: Average magnetisation remaining after a largeangle $\Theta$ pulse. a: data for a $25 \mathrm{~mm}$ long gas sample in the initial coil-shield set (symbols) and expectations for the computed field maps (solid line: $25 \mathrm{~mm}$ sample; dashed line: $10 \mathrm{~mm}$ sample) b: data with the correcting coil and a 10-mm-long liquid sample (symbols). The dashed line is the same as in the upper panel (note the changes in the plot scales). The dotted line correspond to the expectations for the computed map of Fig. $4 \mathrm{~b}$ and a $10 \mathrm{~mm}$ sample; the dash-dotted red line is computed assuming a $0.5 \mathrm{~mm} z$-offset of the correcting coil (see text), which induces the additional field gradient represented by the straight line in Fig. 4 p.

\section{Conclusion}

A local correcting coil nicely compensates the internal field distortion introduced by an efficient shield coil, yield- ing a ten-fold improvement of RF uniformity $( \pm 0.5 \%$ over an extended volume) while hardly affecting the shielding efficiency (the external field remains strongly suppressed, $\sim \div 20$ ). Minor adjustments of the shield geometrical parameters can be used to improve further the shielding efficiency in a selected region of interest with negligible effect on the field uniformity near the axis. Our method for correcting a shielded coil set offers an efficient and flexible solution for low-frequency NMR experiments with very demanding RF homogeneity requirements such as the low temperature studies of Ref. [5] or variable flip angle MRI studies 8 .

\section{Acknowledgements}

This work was supported in part by the FPGG foundation and the IMAGINE project of of the French National Research Agency (V.V.K.), and by the "Research in Paris" programme (M.E.H.).

\section{A Coil construction details}

The three coaxial coils shown in Fig. 1 and used in our experiments were manually wound with $0.15 \mathrm{~mm}$ diameter enamelled wire using the following procedure. The templates reproduced in Fig. 6 were printed on $0.09 \mathrm{~mm}$ thick paper, adjusting print scaling factors to obtain the pattern outer dimensions $W$ and $H$ listed in Table 1 for each of the three coils, together with additional relevant dimensions. The main and shield coils both differ slightly from those of Ref. 4]: the lengths of the elements of the main coil are staggered to avoid wire overlap, and the shield coil is stretched by about $2.5 \%$ in the z-direction, which is expected to yield a slightly improved shielding efficiency (see App. B).

\begin{tabular}{l|lll} 
& Shield & main & corr. \\
\hline$W$ & 99.3 & 79.5 & 75.4 \\
$H$ & 75.1 & 75.1 & 20 \\
$L_{\mathrm{c}}$ & 66.3 & 52.5 & 20 \\
$d_{\mathrm{w}}$ & 31.5 & 25.2 & 23.8 \\
$d_{\mathrm{c}}$ & 32.0 & 25.8 & 24 \\
\hline
\end{tabular}

Table 1: Table of geometrical parameters of the three coils used in the experiments. $W$ : width of the outer box of the coil pattern; $H$ : height of this box; $L_{\mathrm{c}}$ : coil total length; $d_{\mathrm{w}}$ : diameter of the wood former; $d_{\mathrm{c}}$ : average coil diameter.

Each pattern was wound on a cylindrical balsa wood former of diameter $d_{\mathrm{w}}$ covered with a layer of plastic wrap. Straight pins were inserted into the former at the indicated points and series of 10-turn windings were successively wound against them, starting from the innermost loop on each side (photo a in Fig. 7. shield coil). Fluid epoxy glue (Stycast 1266) was used to hold the wires together onto the paper template before removing the pins 


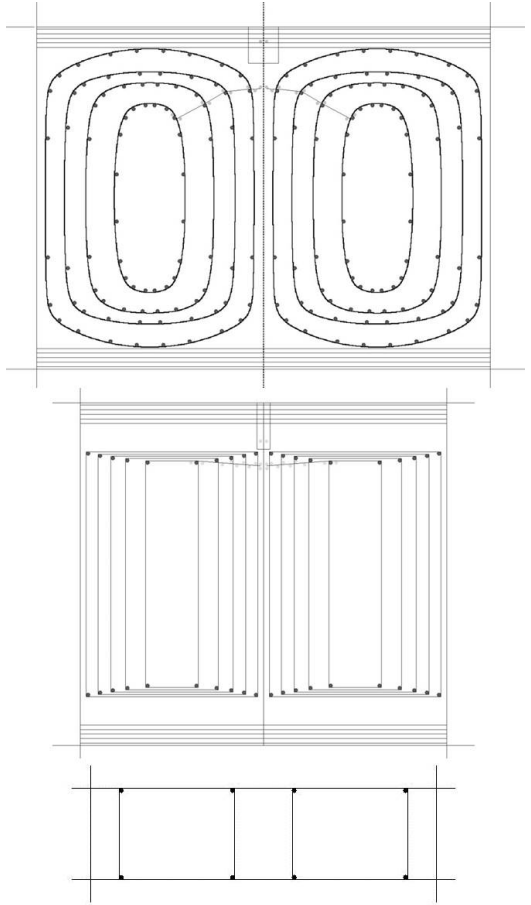

Figure 6: Winding patterns used to make the shield, main, and correcting coils (top to bottom). They can be used to make coils of any size when printed with appropriate scaling factors. The rectangular boxes around the winding patterns are used for accurate positioning of the templates around the wood formers (see Fig. 7).

(photo b in Fig. 7) main coil). The average coil diameter, $d_{\mathrm{c}}$, and the total coil length, $L_{\mathrm{c}}$, resulting from the exact wire layer arrangement were evaluated on the finished coil. The uncertainties on the actual wire positions and

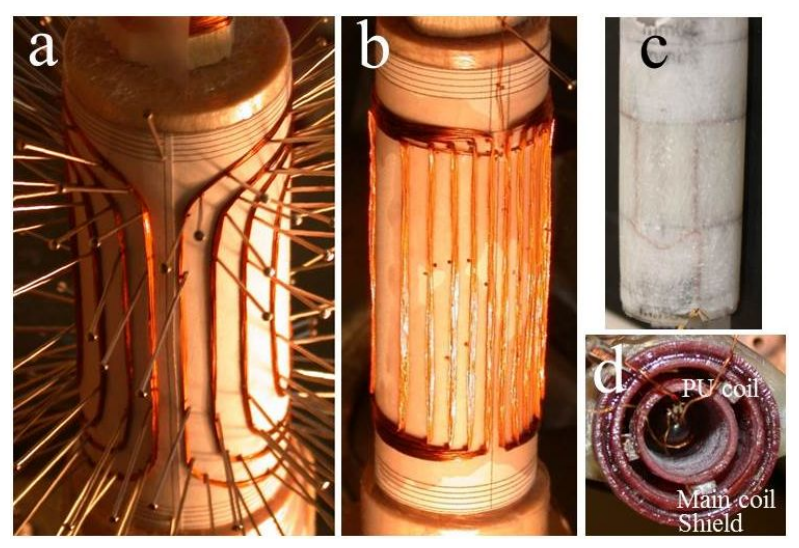

Figure 7: Successive steps in the construction of the coils using the patterns of Fig. 6 (see text). a: initial stage for the shield; b: intermediate stage for the main coil; c: final stage for the correcting coil. $d$ : axial view of the assembled shield-coil set, with an additional inside coil (PU coil), a crossed saddle coil pair used for NMR signal detection, not described here. on the dimensions in the last two lines of Table 1 were evaluated to be of order $\pm 0.3 \mathrm{~mm}$.

Cotton fabric was then wrapped around each coil and impregnated with epoxy glue. The external surface of the epoxy was machined after curing and the balsa core was carved out, leaving a well-protected coil inside an epoxy tube (photo c in Fig. 7; correcting coil). The coils were then assembled and positioned using shims, and reversibly glued with GE180 varnish (photo d in Fig. 7. axial view of an uncorrected transmit / receive coil set).

\section{B Computation of the shielding efficiency}

If the continuous surface currents derived in Ref. 4] (Fig. 6) were used, perfect shielding would be achieved with null field for $\rho>b$ (in cylindrical coordinates). However, when surface currents are replaced by currents in discrete windings, a net field exists outside this imperfect shield. In particular, a remanent dipole field occurs if there is any mismatch in the projected areas of the coil and shield current elements on the yz-plane. (For a saddle-coil element, for example, the dipole moment is $\boldsymbol{m}=8$ Ila $\sin \varphi \hat{\boldsymbol{x}}$ with the notation of Ref. 44.) Here, we present some useful strategies for evaluating and improving the efficiency of discrete current shields, with a focus on the dipolar term.

Figure 8 displays a set of $2 \mathrm{D}$ contour maps of the reduced amplitude of the field, $(\rho / a)^{3} B_{1}(\rho, \phi, z) / B_{1}(0)$, in which the factor $(\rho / a)^{3}$ is introduced to compensate for the trivial dipolar decrease of the field at large distance. Each panel corresponds to a fixed distance $\rho$ from the coil axis. At short distances, the locus of highest field amplitude is found at $z / a \approx \pm 3$ and has a wide angular extension. It switches to the $\mathrm{x}$-axis $(\phi=0, z=0)$ beyond $\rho / a \approx 10$. Hence, although correct at large distance, the evaluation of the shielding efficiency on the $\mathrm{x}$-axis alone does not capture all the features of the shielding imperfections and may overlook experimentally relevant features. Here, we choose to consider the maximum of the field amplitude at fixed $\rho, \beta_{\max }(\rho)=\max \left[B_{1}(\rho, \phi, z)\right] / B_{1}(0)$, which provides a worst-case assessment of the shielding efficiency.

Figure $9 a$ displays the variation of $(\rho / a)^{3} \beta_{\max }$ with $\rho$ for the main coil alone (squares), the coil-shield set (circles), and the corrected coil (triangles). The solid lines (with only one symbol mark) correspond to the coils of Ref. 4, the open symbols to the coils actually used in our experiment (see App. A. There is no significant difference for the fields of the main coils outside the volume of the shield (the hatched box in the figure).

The addition of a 4-loop shield coil to the main coil (red line or circles) significantly decreases the external field, with a reduction factor of order $15-20$ beyond $\rho / a \approx 3$. Each asymptotic value in Figure 9 corresponds to a net dipolar field. This originates from using the stream func- 


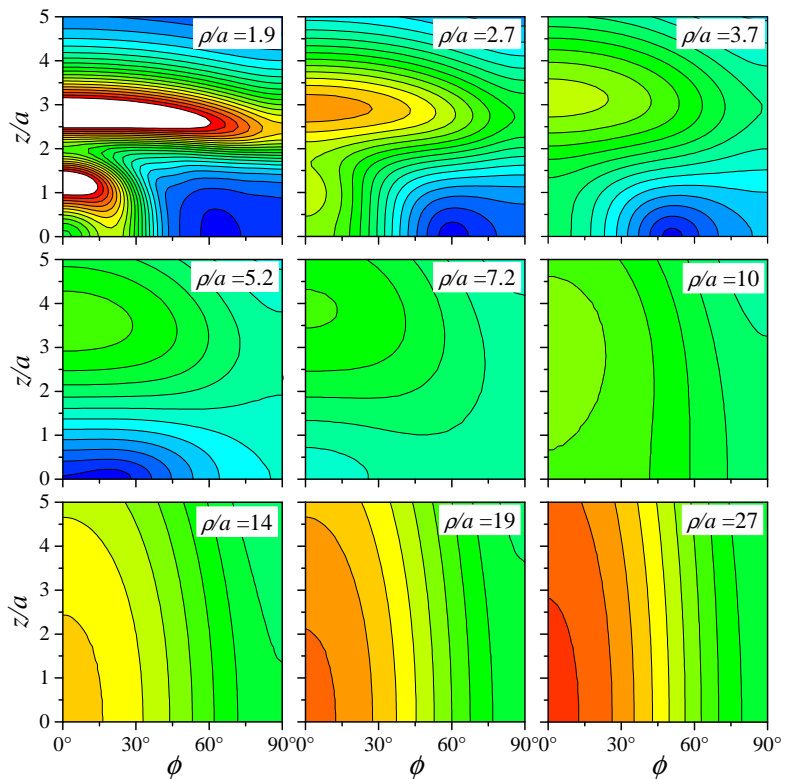

Figure 8: 2D contour plots of $(\rho / a)^{3} B_{1}(\rho, \phi, z) / B_{1}(0)$ computed for increasing values of $\rho / a$ (indicated on each panel). $\phi=0$ corresponds to the $\mathrm{x}$-axis direction, which is that of $\mathbf{B}_{1}(0)$ (see Fig. 1). The common scale of all panels ranges from 0 to 0.25 (from blue to red), with 20 contour lines separated by steps of $1.25 \times 10^{-2}$. Computations are made for the coil-shield set of Ref 4 .

tion method to define the current elements: this convenient recipe provides a good but imperfect approximation of the continuous currents. In particular, the dipolar term in the multipolar field expansion is found to be $2.37 \%$ too small. Figure 9b displays examples of variations of $\beta_{\max }$ for slight distortions of the shield coil in the coil-shield set of Ref. 4] (uniform stretching of the shield along the z-axis by 1 to $3 \%$ ). As expected, $\beta_{\max }$ can be significantly improved at large $\rho$ when the dipolar term is cancelled, but this is at the expense of the shielding efficiency at intermediate distances $(\rho / a<10)$. Additional calculations show that using more loops for the shield (up to 8 pairs instead of 4 ) only reduces $\beta_{\max }$ by a factor 2 , and performing different kinds of small geometrical loop shape distortions provide very similar results (not displayed).

The addition of the correcting coil, for which the dipolar term is less than $2 \%$ of the main coil, only weakly modifies the amplitude of the external field: this is the difference between the red curve and the and blue curve or circles and triangles, depending on the coil set. For the coil set used in the experiment, the addition of the correction coil actually improves the computed shielding efficiency at intermediate distances $(2<\rho / a<10)$ without penalty at large $\rho$ (the near-identical values of $\beta_{\max }$ in Fig. 9p actually result from different field maps). However, the actual benefit is limited, in fact of the same order as the changes in the computed values resulting from the expected geometrical imperfections of our coils.
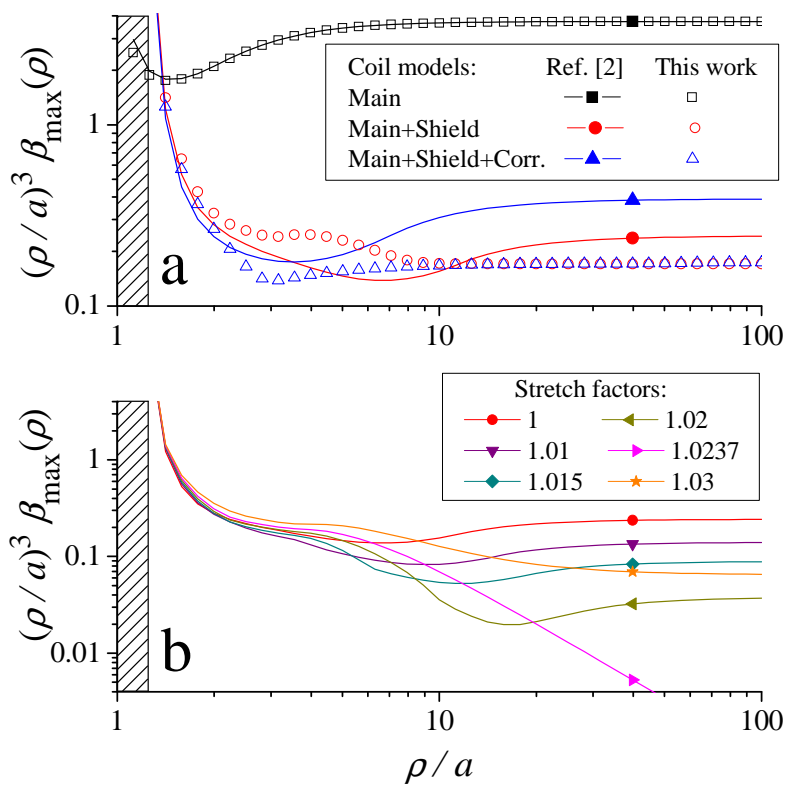

Figure 9: Plots of the computed maximum reduced field amplitudes (see text) as functions of $\rho / a$ (hatched boxes: inner volume of the shield, $\rho<b$ ). a: Comparison of the coils of Ref. [4] (solid lines) and of this work (open symbols, see legend). b: Coil-shield set of Ref. 4], but with the shield stretched in the $z$ direction (see legend) so as to vary the compensation of dipolar contributions of the main and shield coils.

\section{RF map reconstruction}

The reconstruction of the $1 \mathrm{D}$ map of the RF field, $B_{1}(z)$, makes use of the two $1 \mathrm{D}$ images, $M_{1}(z)$ and $M_{2}(z)$, obtained by Fourier transforms of spin echo signals for rotations of average angles $\Theta_{1}$ and $\Theta_{2}=\Theta_{1}+\pi / 2$, respectively (Sec. 3.1. Fig. 33). If the field map is written as $B_{1}(z)=[1+\delta(z)] \bar{B}_{1}$, in which $\bar{B}_{1}$ is averaged over the sample volume, the local rotation angle is:

$$
\theta_{1,2}(z)=[1+\delta(z)] \Theta_{1,2} .
$$

The out-of-phase demodulated signal contributions $S_{1,2}(z)$ arising from the isochromat located at position $z$ just after the $\Theta$ pulses are given by:

$$
\begin{aligned}
& S_{1}(z)=\Sigma(z) \sin \left[2 \pi X_{\text {rot }}(1+\delta(z))\right] \\
& S_{2}(z)=\Sigma(z) \sin \left[2 \pi\left(X_{\text {rot }}+1 / 4\right)(1+\delta(z))\right],
\end{aligned}
$$

where $X_{\text {rot }}=\Theta_{1} / 2 \pi$ is the number of turns corresponding to the average angle $\Theta_{1}$, and $\Sigma(z)$ is a scaling factor involving magnetisation density and detection sensitivity; in our experiments, it weakly depends on $z$ and has a uniform sign. Simple trigonometric relations can be used to link $\delta(z)$, hence $B_{1}(z)$, to the ratio $S_{1} / S_{2}$ :

$$
\left[\cos \frac{\pi \delta}{2} \cot 2 \pi X_{\text {rot }} \delta-\sin \frac{\pi \delta}{2}\right]^{-1}=S_{1} / S_{2} .
$$

The $1 \mathrm{D}$ images $M_{1,2}$ normally differ from $S_{1,2}$ merely by $z$-dependent phase factors that build up until the end 
of the refocussing $\pi$ pulse. For perfectly identical gradient pulses and acquisition conditions, the same phase factor $\exp i \Phi(z)$ would appear in both images and would drop out of the ratio $M_{1} / M_{2}$. Equation 4 can be solved replacing $S_{1} / S_{2}$ by $\Re\left[M_{1} / M_{2}\right]$, where taking the real part removes the small unwanted contributions arising from noise and imperfect repetitions of gradient sequences. Figure 10 displays the variation of the left hand side of Eq. 4 with $\delta$; for any value of $S_{1} / S_{2}$, a first solution is found for $|\delta| \leq \delta_{1}$, a second one for $\delta_{1} \leq|\delta| \leq 1 / 2 X_{\text {rot }}$.
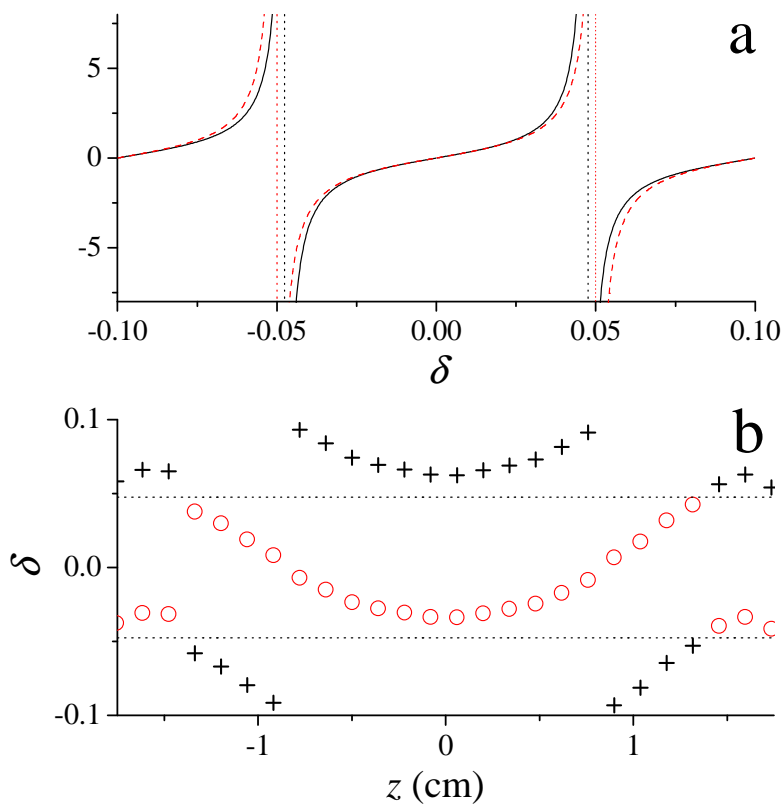

Figure 10: a: Plot of the left hand side of Eq. 4 (black solid line) and of an approximate form (red dashed line) for $X_{\text {rot }}=5$. Each form yields a single solution for $|\delta|<$ $\delta_{1}$, between its vertical asymptotes (dotted lines). $\delta_{1}=$ 0.0476 for Eq. 4 instead of 0.05 for the approximate form. b: First (circles) and second (crosses) solutions of Eq. 4 (see text). The dotted lines $\left(\delta= \pm \delta_{1}\right)$ correspond to the asymptotes in the upper panel.

Figure 10p displays the two solutions of Eq. 4 numerically obtained for the measurements shown in Fig. 4. Over most of the sample length, $|\delta| \leq \delta_{1}$ is the physically relevant solution since the volume average of $\delta$ over the sample is zero. However, at the sample ends, the jump of the value of $\delta$ is unphysical and the second solution must be chosen.

This was directly verified using a determination of $\Phi(z)$ obtained from an additional 1D image following a $\pi / 2$ rotation $\left(X_{\text {rot }}=1 / 4\right.$, yielding a near-uniform transverse magnetization) with the same imaging sequence. The signal contributions $S_{1,2}$ were deduced from the images using:

$$
\left.S_{1,2}=\Re\left[M_{1,2}(z) \exp -i \Phi(z)\right)\right] .
$$

Equation 4 again yields two solutions for each value of $z$ (with $|\delta| \leq 1 / 2 X_{\text {rot }}$ ), but only one satisfies Eqs. 2 and 3 the same one that was chosen in the discussion of Fig. $10 \mathrm{p}$.
Figure 10p clearly shows that the choice $X_{\text {rot }}=5$ is well suited to mapping the RF field of our uncorrected coil set, with good sensitivity and manageable aliasing.

This RF map reconstruction can be used to additionally show that the solution of the approximate form of Eq. 4 $\left(\tan \theta_{1}(z)=\Re\left[M_{1}(z) / M_{2}(z)\right]\right.$, mentioned in Sec. 3.1) overestimates the exact solution by $5 \%$ at most in the conditions of our experiment. This approximation was used for simplicity to evaluate the error bars plotted in Fig. 4 from the noise in the $1 \mathrm{D}$ images. The uncertainties on $M_{1}$ and $M_{2}$ were inferred from data recorded under the same conditions with an unpolarised sample.

\section{References}

[1] C. E. Hayes, W. A. Edelstein, J. F. Schenck, O. M. Mueller, et al. 'An efficient, highly homogeneous radiofrequency coil for whole-body NMR imaging at 1.5T.' J. Magn. Reson., 63(3):622-628 (1985)

[2] G. Giovannetti, R. Francesconi, L. Landini, M. F. Santarelli, et al. 'Conductor geometry and capacitor quality for performance optimization of low-frequency birdcage coils.' Concepts Magn. Reson., 20B(1):9-16 (2004)

[3] A. M. Coffey, M. L. Truong, and E. Y. Chekmenev. 'Low-field MRI can be more sensitive than high-field MRI.' J. Magn. Reson., 237(0):169 - 174 (2013).

[4] C. P. Bidinosti, I. S. Kravchuk, and M. E. Hayden. 'Active shielding of cylindrical saddle-shaped coils: Application to wire-wound RF coils for very low field NMR and MRI.' J. Magn. Reson., 177(1):31-43 (2005)

[5] M. E. Hayden, E. Baudin, G. Tastevin, and P.-J. Nacher. 'NMR Time Reversal as a Probe of Incipient Turbulent Spin Dynamics.' Phys. Rev. Lett., 99(13):137602 (2007).

[6] M. Himbert, J. Dupont-Roc, and C. Lhuillier. 'Spindiffusion coefficient in gaseous helium between 1 and 0.5 K.' Phys. Rev. A, 39:6170-6177 (1989).

[7] R. Tycko. 'Broadband Population Inversion.' Phys. Rev. Lett., 51:775-777 (1983).

[8] A. Ouriadov, W. Lam, and G. Santyr. 'Rapid 3$\mathrm{D}$ mapping of hyperpolarized $3 \mathrm{He}$ spin-lattice relaxation times using variable flip angle gradient echo imaging with application to alveolar oxygen partial pressure measurement in rat lungs.' Magnetic Resonance Materials in Physics, Biology and Medicine, 22(5):309-318 (2009). 